\section{Rapport sur la session ordinaire de la Chambre médicale suisse des 21 et 22 juin 2000 , à Berne}

\author{
F.-X. Deschenaux, secrétaire général de la FMH
}

Cette session de la Chambre médicale est à marquer d'une pierre blanche pour être la première à être composée conformément aux nouveaux statuts de la FMH.

La présente session a réservé aux 196 délégués recensés un ordre du jour quasi titanesque, avec 89 propositions déposées au début de la séance, comprenant comme pièces de résistance, outre les affaires administratives usuelles (rapport annuel, comptes de la FMH pour l'exercice écoulé et budget pour l'exercice suivant), les élections pour la période administrative 2000-2003, la nouvelle systématique des titres de spécialiste et les programmes de formation, ainsi que des discussions nourries autour de l'obligation de contracter et des derniers développements du TarMed. Ces deux derniers objets ont pris comme on peut bien l'imaginer une résonance toute particulière sous l'effet des interventions récentes - ce qui ne procède certainement pas du hasard - du Département fédéral de l'intérieur et de sa cheffe.

Les décisions sont restituées dans le présent procès-verbal selon l'ordre séquentiel figurant sur la liste des tractanda.

\section{Communications et constitution du Bureau}

La séance est ouverte à 9h45, alors que le quorum de 100 délégués est largement atteint.

Les scrutateurs proposés par le président (Drs A. Sury, B. Hug, A. Bacchetto, A. Froidevaux, C. Ehrler et $\mathrm{M}$. Hug) sont acceptés tacitement par les délégués; il s'agissait des scrutateurs de la première journée. Pour la seconde journée, les scrutateurs proposés en début de séance par le président et acceptés tacitement par les délégués furent les Drs Z. Schneider, C. Ehrler, A. Kubli Bauer, M. Schibli, M. Kondo Oestreicher et C. Brunner.

Le Bureau chargé de dépouiller les bulletins de vote pour les élections administratives 2000-2003 est constitué par des collaborateurs du Secrétariat général placés sous la direction de M. C. Kreyden, ce, afin que les délégués-scrutateurs ne soient pas privés de participer aux débats et aux prises de décision se poursuivant pendant les opérations de dépouillement. Ce mode d'opérer appartient à une tradition bien établie qui n'est pas remise en question par les délégués.

Le président $\mathrm{H}$. $\mathrm{H}$. Brunner tient à préciser à l'intention du procès-verbal, pour maintenant et le futur, la réglementation à respecter en ce qui concerne les personnes accompagnantes. Les organisations de base (sociétés cantonales de médecine [ci-après: SCM]), l'Association des médecins-assistants et chefs de clinique (ASMAC) ont jusqu'ici été autorisées à se faire accompagner d'un conseiller non-médecin qui a le droit de prendre la parole jusqu'à ce qu'une motion d'ordre le lui dénie. Cet usage est maintenu, mais le CC, resp. le président se réserve le droit d'exiger que les éventuels accompagnants non-médecins de sociétés de discipline médicale (ci-après: SDM) siègent non pas dans la salle, mais dans les tribunes, vu la place restreinte découlant du grand effectif des délégués. A part cela, le CC se réserve toujours le droit d'inviter aux séances de la Chambre médicale, pour le traitement d'un objet particulier, telle ou telle personne extérieure dont la présence se révélera nécessaire.

Compte tenu de l'impressionnante liste des objets à traiter lors de la présente session, le président laisse entendre que l'on va vraisemblablement siéger la première journée jusqu'aux alentours de 18h30, l'heure de clôture de la séance du lendemain allant dépendre de la liquidation de l'ordre du jour.

Conformément au nouveau Règlement d'exécution de la FMH du 8 avril 1999 prenant lui-même appui sur l'article 35, al. 2, des nouveaux statuts, il ne sera dressé dorénavant qu'un procès-verbal de décisions, enrichi ici ou là de quelques explications supplémentaires pour autant que besoin.

Le président constate en outre que la convocation et la liste des tractanda, y compris la publication dans le Bulletin des médecins suisses (ci-après: BMS), ont été communiquées dans les délais. Toutes les propositions parvenues jusqu'à lundi soir ont été traduites.

A l'invitation du président, l'administrateur de FMH Services, M. M. Baumgartner, explique que les nouveaux statuts de FMH Services permettent également à des non-membres de la FMH de faire partie de cette société, raison pour laquelle la tenue traditionnelle d'une assemblée générale des délégués de FMH Services avant la Chambre médicale ordinaire n'a plus lieu d'être. L'assemblée des délégués de FMH Services est désormais remplacée par une assemblée universelle qui prendra ses décisions sous forme de votation générale pour laquelle les membres recevront le matériel de vote prochainement.

Le président conclut ce premier point de l'ordre $\mathrm{du}$ jour en rendant hommage aux consœurs et confrères décédés au cours de l'exercice écoulé. Il tient à évoquer plus particulièrement la mémoire du Dr Jürg Ammann, ancien président de la FMS, disparu récemment et dont il met en exergue les grands mérites qu'il s'est acquis pour son engagement exceptionnel pour la cause de ses confrères chirurgiens. Il relève que sa lutte a été loin de s'épuiser dans la seule défense de leurs rémunérations, car son action 
visait beaucoup plus haut et s'inscrivait dans ses efforts pour enrayer la dévaluation de leur statut professionnel. Il mérite en ce sens la reconnaissance de l'ensemble du corps médical.

A l'invitation du président, l'assemblée se lève et observe quelques instants de silence à la mémoire de tous nos membres disparus.

\section{Rapport annuel 1999/2000}

Le rapport annuel, élaboré par le président de la FMH, les membres du CC responsables de ressorts, le secrétaire général et les divers responsables de division et de services au sein du Secrétariat général, a été publié dans le BMS n² 23 du 7 juin 2000.

Le président le complète par quelques remarques rétrospectives et prospectives pour la période administrative qui s'ouvre à compter d'aujourd'hui.

La parole n'est pas demandée après ce tour d'horizon présidentiel et le rapport annuel est alors approuvé sans opposition.

\subsection{Comptes 1999 de la FMH}

\subsubsection{Approbation}

Le bilan et les comptes annuels de la FMH pour l'exercice 1999 et le rapport de vérification de la fiduciaire PriceWaterhouseCoopers qui y était joint sont approuvés à une quasi unanimité, avec une abstention. L'excédent de dépenses de Fr. 77 744.98, sensiblement inférieur aux quelque Fr. $429000 .-$ qui avaient été prévus par le budget, est mis à la charge du capital propre qui s'élève ainsi, au 31 décembre 1999, à Fr. 4450873.69.

Dans la foulée, le bilan et les comptes d'exploitation au 31 décembre 1999 du Fonds d'assistance de la FMH, ainsi que le bilan et les comptes d'exploitation au 31 décembre 1999 du Fonds pour l'encouragement de la formation postgraduée des médecins dans les pays en développement sont approuvés simultanément.

\subsubsection{Octroi de la décharge au Comité central}

La décharge aux organes responsables, pour leur gestion, est votée à une très forte majorité, avec une abstention.

Proposition du Dr R. Streit, Société des médecins du canton de Berne, ad point 3.1

Cette proposition, qui reçoit le soutien inconditionnel du Dr M. Battaglia, au nom de l'ASMAC, a le libellé suivant: "Se fondant sur les principes de la comptabilité analytique, le Comité central est chargé de démontrer, à la Chambre médicale 2001, comment les coûts et les recettes sont répartis par unité de gestion. Il s'agit aussi d'indiquer le personnel occupé par centre de charges".

Le président H. H. Brunner, au nom du CC, accepte ladite proposition.

\subsection{Budget 2001 de la FMH}

Le budget présenté à la Chambre médicale pour l'année 2001 comporte deux volets: le budget général pour un total de recettes de Fr. 12755000 .- ainsi qu'un total de dépenses de Fr. $12684000 .-$, soit un excédent de recettes de Fr. $71000 .-$, d'une part, et des contributions spéciales et limitées dans le temps pour financer des activités particulières, d'autre part. Tel que présenté, le budget général est fondé sur une cotisation centrale de Fr. 480.- pour les membres des catégories 1 (médecins en pratique privée) et 2 (médecins salariés exerçant une fonction dirigeante), de Fr. 240.- pour les membres des catégories 3 (médecins salariés sans fonction dirigeante et ne se trouvant pas en formation postgraduée FMH) et 4 (médecins en formation postgraduée) et enfin de Fr. 120.pour les membres des catégories 5 (médecins domiciliés et exerçant à l'étranger) et 6 (membres temporairement sans activité médicale).

Sur proposition du CC acceptée tacitement par la Chambre médicale, on vote séparément sur le budget général et sur les contributions particulières.

Le budget général ne donne pas lieu à discussion. Il est accepté à la majorité moins une voix et trois abstentions.

Les contributions spéciales donnent en revanche lieu à une discussion nourrie dont nous résumons ci-après les décisions. La somme des contributions spéciales initialement prévues par le CC s'élevait à Fr. 120.- pour tous les membres et était censée financer les diverses tâches suivantes: Centre suisse de pharmacovigilance: Fr. 5.-, pour la dernière fois en 2001; contribution unique de Fr. 20.- en 2001 afin de financer le développement des éditions EMH; "Abonnement HIN pour tous", à prélever en 2001, 2002 et 2003, au maximum Fr. 50.-; "Assistanat au cabinet médical" Fr. 25.- pour les catégories de membres 1, 2, 3 et 4; enfin Fr. 20.- pour la fin des travaux et l'introduction du TarMed pour les catégories de membres 1, 2, 3 et 4 .

A ceux qui, comme le Dr R. Streit (BE), auraient souhaité que l'on procède le plus possible à demander les fonds nécessaires en les intégrant dans le budget général, le président justifie le principe de contributions spéciales par un souci de transparence et par le regroupement du financement de tâches limitées dans le temps. Quant aux représentants de l'ASMAC (Drs M. Battaglia et S. Stöhr), tout en exprimant des réserves sur certains objets (HIN en particulier), ils ne contestent pas le principe d'un financement solidaire mais ils proposent que celui-ci soit mieux modulé, dans la même proportion que celle qui a cours entre les différentes catégories de cotisations. Cela dit, le financement de ces tâches n'est contesté par personne, certains délégués rompant même une lance pour elles, en particulier pour le HIN.

Le président $\mathrm{H}$. H. Brunner est alors amené à proposer une solution de compromis au nom du CC. Il propose la perception d'une contribution spéciale soit de Fr. 100.- pour tous (variante I), soit de Fr. 70.- pour les catégories de cotisations 3 et 4 et de Fr. 110.- pour les catégories 1 et 2 (variante II). La variante qui l'em- 
portera sera alors opposée, dans un vote définitif, à la proposition de l'ASMAC. Ce mode de faire est accepté et le Dr P.-A. Schneider, de l'Association des médecins du canton de Genève (AMG), qui avait soumis une proposition demandant que la contribution pour l'assistanat au cabinet médical soit réduite de Fr. 25.- à Fr. 10.- retire sur ces entrefaites sa proposition. Le président précise, avant le vote, que les sommes récoltées au titre des contributions spéciales seront attribuées aux divers objets par décision du CC.

Dans un premier vote très serré, la variante I l'emporte sur la variante II par 76 voix contre 75. Dans un second vote opposant la variante I à la proposition de l'ASMAC, la variante I l'emporte par 117 voix contre 49, avec 3 abstentions.

On ne quittera pas ce chapitre sans évoquer encore ici une suggestion du président de la Société de médecine du canton de St-Gall, le Dr R. Urscheler, demandant que l'on organise une fois encore, lors de la prochaine séance de la Conférence des présidents, un sondage auprès des SCM concernant l'encaissement des cotisations de la FMH, pour savoir si ce sont les SCM qui doivent y procéder ou le Secrétariat général de la FMH. Dont acte.

\section{Elections pour la période administrative 2000-2003}

4.1 Fixation du nombre de membres du CC pour la prochaine période administrative Cette question met en compétition des propositions contradictoires. D'une part, l'ASMAC et la Société médicale de Bâle-Ville présentent des propositions tendant à ce que l'effectif du CC soit maintenu à 11 membres; D'autre part, le Dr A. Haefeli, président du VEDAG, avait initialement présenté une proposition demandant que les deux sièges alémaniques devenus vacants au sein du CC ne soient pas remplacés, dans le sens en quelque sorte d'un moratoire. Le Dr A. Haefeli retira cependant sa proposition au cours des délibérations, compte tenu de ce qu'un siège doit être statutairement réservé à l'Association des médecins principaux d'hôpitaux de Suisse (AMPHS) et de ce que même ceux qui plaident aujourd'hui pour un CC de 11 membres ne s'opposent pas au principe d'une réduction ultérieure, pour autant que le CC présente d'ici 2001 un nouveau concept pour la direction de la FMH. Le Dr A. Haefeli se range donc sans restriction à la proposition du Dr R. Streit présentée ci-après sous point 5 et déclare que le volet de la proposition du VEDAG, en tant qu'il ne concerne pas le moratoire pour la nouvelle période administrative, devra être discuté sous point 5/changements des statuts.

D'autres délégués soutiennent d'ores et déjà un effectif réduit à 9 membres. Il s'agit donc de départager les deux thèses en présence. Au vote, les délégués décident, par 125 voix contre 48 avec 4 abstentions, d'en rester pour la période administrative 2000-2003 à la composition d'un CC de 11 membres.

\subsection{Election du Comité central de la FMH}

Confirmation des membres actuels

Bulletins rentrés

Bulletins valables

Majorité absolue

Sont réélus:

VEDAG

Dr Hans Heinrich Brunner (LU) 165 voix

Dr Max Giger (ZH)

Dr Reto Laetsch (SG)

Dr Ursula Steiner-König (BE)

150 voix

156 voix

155 voix

SMSR

Dr Claude Aubert (GE) 166 voix

Dr Yves Guisan (VD)

152 voix

Dr Marie-Christine Peter-Gattlen (VS)

158 voix

ASMAC

M. le Dr Ludwig-Theodor Heuss (BS)

167 voix

Elections pour les sièges à repourvoir ORDINE (OMCT)

Bulletins rentrés

Bulletins valables

174

Majorité absolue

Est élu:

Dr Franco Muggli (TI)

174 voix

AMPHS

Bulletins rentrés

Bulletins valables

Majorité absolue

Est élue:

Dr Verena Briner, p.-d. (LU)

94 voix

Ont recueilli en outre des suffrages:

Dr Yves Guisan (VD)

Prof. Werner Straub (BE)

Suffrage isolé

58 voix 15 voix 1 voix

Siège restant

Bulletins rentrés

Bulletins valables

161

Majorité absolue

Est élue:

Dr Susanna Stöhr (BS)

83 voix

Ont recueilli en outre des suffrages:

Dr Catherine Wehren (BS)

Dr Ludwig-Theodor Heuss (BS)

76 voix 2 voix

4.3 Election du président de la FMH

Bulletins rentrés

Bulletins valables

Majorité absolue 
Est élu:

Dr Hans Heinrich Brunner

152 voix

Ont recueilli en outre des suffrages:

Dr Claude Aubert

Dr Yves Guisan

Dr Ludwig-Theodor Heuss

3 voix

5 voix

3 voix

\subsection{Election des deux vice-présidents de la FMH}

Elections à mains levées

Donnant suite à une suggestion venant de l'assemblée, le président $\mathrm{H}$. $\mathrm{H}$. Brunner met tout d'abord aux voix la question de savoir si les délégués acceptent qu'à partir de maintenant, les élections soient effectuées à mains levées. Les délégués approuvent ce mode de faire par 147 voix contre 19 et 3 abstentions, soit à une majorité dépassant les $3 / 4$ (127) requis par les statuts.

Participent à l'élection des vice-présidents: 170 délégués

Sont élus:

Dr Ursula Steiner-König (nouvelle)

152 voix

Dr Yves Guisan (ancien)

143 voix

4.5 Election du président de la Commission de la formation postgraduée et continue (CFPC)

Participent à l'élection du président de la CFPC: 159 délégués.

Est élu:

Dr Max Giger (nouveau)

150 voix

\subsection{Election de la Commission des finances de la FMH}

Il est procédé à cette élection «in globo», soit pour le renouvellement des deux membres sortants, les Drs 0 . Kappeler (TG) et A. Sury (TI), ainsi que pour le nouveau membre proposé par la SMSR, le Dr Pierre Guillaume (FR). Ils sont tous trois élus par 171 voix.

\subsection{Election du Bureau du Conseil suisse de déontologie (CSD)}

Il est également procédé à cette élection "in globo", par acclamations, aucun autre candidat que ceux proposés par le VEDAG, la SMSR et l'ORDINE n'ayant été présentés. Il s'agit du Dr Sergio Luisoli (TI), ancien, du Prof. Markus Heinz Knoblauch (ZH), nouveau, et du Dr Dominique Haefeli, (NE), nouveau.

NB: L'ancien et les nouveaux membres n'ayant pas encore eu l'occasion de se rencontrer, l'élection du président du CSD est différée jusqu'à la prochaine session de la Chambre médicale.

4.8 Comité consultatif pour les négociations TarMed Le CC avait proposé à la Chambre médicale de constituer un comité consultatif de 10 membres, soit 4 pour la Foederatio Medicorum Scrutantium (FMS), 4 pour le Collège de médecine de premier recours (CMPR) et 2 pour la Foederatio Medicorum Curantium (FMC).

Une proposition conjointe de la Société suisse de psychiatrie et psychothérapie et de la Société suisse de psychiatrie et psychothérapie d'enfants et d'adolescents demande que la composition de ce comité consultatif soit portée à 12 membres afin que deux sièges supplémentaires puissent y être réservés à la SSPP.

Mise au vote, la proposition des psychiatres est acceptée par 138 voix contre 21 et 8 abstentions.

Sur ces entrefaites, la Chambre médicale prend connaissance des propositions de la FMS, du CMPR, de la FMC et de la SSPP et les accepte tacitement et sans autre contre-propositions. Siégeront donc dans ce comité consultatif de 12 membres:

Pour la FMS: les Drs Beat Meister (BE), Alexander Eijsten (ZH), Walter P. Gartenmann (ZH) et Roberto Tartini (ZH). Quatre remplaçants ont également été prévus par la FMS en cas d'empêchement des élus: Me François A. Bernath et les Drs Christoph Krayenbühl, Rolf Steiner et Josef Hug;

Pour le CMPR: les Drs Christoph Franke (SG), Niklaus Hasler (ZH), Juerg Pellaton (ZH) et Emile Simon (VD);

Pour la FMC: les Drs Markus Heitz (ZH) et Andreas Wüest (ZH);

Pour la SSPP: les Drs Heiner Lachenmeier (ZH) et Urs Fromm (AG).

Avant de quitter le chapitre des élections, le président $\mathrm{H}$. H. Brunner tient à rendre hommage aux trois membres du CC qui nous quittent à la fin de la période administrative écoulée et à les remercier, en relevant leurs mérites et les tâches accomplies au service de la FMH et de l'ensemble du corps médical au cours de nombreuses années d'activité. Nous reviendrons ultérieurement sur le sujet dans un article du BMS. Chacun des membres démissionnaires s'adresse ensuite personnellement à l'assemblée et recueille les applaudissements des délégués.

\section{Changements des statuts}

Le Dr A. Haefeli, au nom du VEDAG, reprend ici l'idée déjà évoquée dans une proposition ad ch. 4.1 (voir supra) par laquelle le Comité central est invité à présenter à la Chambre médicale un projet de restructuration et de révision de sa manière de travailler, en tenant compte de la réduction du nombre de membres, de la représentation appropriée des régions linguistiques, de la non-éligibilité des représentants de groupes d'intérêts et de la divulgation de liens potentiellement problématiques, de l'augmentation du taux de professionnalisme, du rôle plus actif du CC et de son président lors du choix des candidats et enfin de la préparation des candidats sur une période plus longue à assumer leur fonction compte tenu du changement d'activité professionnelle. Calendrier prévu: première version à présenter à la Chambre médicale de juin 2001 et concept détaillé en 2002, afin que les candidats au CC de la prochaine période administrative puissent être préparés suffisamment tôt. 
Au vote, la proposition $d u$ VEDAG, à laquelle le CC ne s'est pas opposé, est acceptée par 132 voix contre une, avec 17 abstentions.

Une proposition $n^{\circ} 3$ ad point 5 de l'ordre du jour, présentée au nom de la Société des médecins du canton de Berne, par le Dr R. Streit vise en substance les mêmes objectifs que ceux de la proposition du VEDAG, mais demande en plus que «un comité élargi aux compétences précises [vienne] se placer entre le petit comité et la Chambre médicale».

Après un bref débat dans lequel les intervenants expriment en majorité leur scepticisme à propos d'une telle institution, on passe au vote. La proposition du Dr R. Streit est rejetée par 102 voix contre 49, avec 21 abstentions.

Une proposition conjointe de la SSMG et de la $S S M I n^{\circ} 1$ ad point 5 de l'ordre du jour, vise à ce que "la FMH [soit] chargée de formuler des critères appropriés, garantissant la proportionnalité de la répartition des sièges des sociétés de discipline médicale à la Chambre médicale en conformité avec les nouveaux statuts de la FMH».

Cette proposition est acceptée par 134 voix contre 25, avec 8 abstentions.

Ce résultat amène Mme le $\mathrm{Dr} \mathrm{A}$. Kubli à retirer la proposition $n^{\circ} 2$ ad point 5 présentée conjointement par les deux sociétés de psychiatrie et de psychothérapie (adultes/enfants et adolescents) et qui poursuivait le même objectif.

\section{Formation postgraduée}

6.1 Nouvelle systématique des titres de spécialiste/ programmes de formation

\subsubsection{Révision des titres de spécialiste}

Avant d'entrer dans le détail des délibérations et des décisions, le Dr R. Salzberg, président sortant de la CFPC, tient à situer le cadre du débat, des enjeux et des décisions. Il rappelle qu'en 1994, devant la profusion des nouveaux titres, un moratoire fut décrété. Il en est résulté une révision de la Réglementation sur la formation postgraduée (RFP) en 1997 qui a conduit à la création de nouveaux titres de spécialiste et à la création de formations approfondies, d'attestations de formation complémentaire et de certificats d'aptitude technique censés remplacer et intégrer les anciennes sous-spécialités abolies. Depuis lors, de nouveaux titres de spécialiste ont été créés ainsi que des formations approfondies. La Chambre médicale a développé un usage prévoyant que la formation postgraduée devait durer au maximum 6 ans, que l'on allait se montrer restrictif avec la création de nouveaux titres et que ces derniers devraient compter au minimum avec 3 ans de formation postgraduée spécifique dans la spécialité. Il fut en outre décidé d'adopter un modèle selon lequel le titre de spécialiste +3 ans de formation complémentaire dans la discipline pourrait conduire à une formation approfondie (ex.: la néonatologie).
Nous avons à discuter aujourd'hui de deux genres de problèmes qui ne s'inscrivent pas dans ce concept, celui de la neuroradiologie et celui des anciennes sous-spécialités de la pédiatrie. L'intervenant rappelle quelles sont les considérations de principe à retenir pour les décisions à prendre par la Chambre médicale: un titre de spécialiste sert à attester qu'une formation postgraduée structurée et contrôlée a été accomplie conformément au règlement dans une discipline clinique ou non clinique de la médecine, tandis que, comme son nom l'indique, une formation approfondie atteste que la formation postgraduée a été poussée plus avant dans un domaine particulier d'une discipline. Les groupements responsables de ces formations approfondies sont représentés dans la CFPC et peuvent y faire valoir leur desiderata; en revanche, ces dits groupements ne sont pas représentés comme tels à la Chambre médicale et doivent alors passer par leur société mère pour y présenter leurs demandes (ex.: les néonatologues doivent en mander la Société suisse de pédiatrie, etc.). Par ailleurs, nous devons tenir compte de la pratique suivie en la matière par les médecins de la Communauté européenne et examiner la façon dont ces domaines de spécialisation y sont réglés. Enfin, chaque société de discipline qui gère une spécialité a l'obligation de maintenir à jour son programme de formation postgraduée et de l'adapter à l'évolution de la médecine, elle doit organiser un examen pour l'obtention du titre de spécialiste et garantir le perfectionnement et la qualité des prestations de ses membres.

Il appartient maintenant à la Chambre médicale de décider si elle veut créer une spécialité ou une formation approfondie, alors que la fixation des programmes est du ressort du CC. Si une société de spécialistes soumet un programme pour la création d'un titre de spécialiste et qu'il y a unité de vues au sein de la CFPC, on ne discute pas sur cette proposition. En revanche, si des propositions sont présentées à propos de ces programmes, on en débat alors à la Chambre.

On passe alors à la discussion de détail.

Dans une proposition $n^{\circ} 1$ ad point 6 , le Dr M. Battaglia, au nom de l'ASMAC, propose une modification de l'ordre du jour consistant en ce que l'on traite tout d'abord sous point 6.1. la révision de la RFP (prévue dans l'ordre du jour sous point 6.2) et que l'on ne traite qu'après tous les objets relatifs à la nouvelle systématique des titres de spécialiste/programmes de formation (prévus sous point 6.1). Le Dr R. Salzberg, au nom du CC, s'oppose à cette modification des règles du jeu.

Au vote, la proposition de l'ASMAC est rejetée par 124 voix contre 30 , avec 10 abstentions.

On poursuit donc les délibérations conformément à l'ordre du jour prévu par le CC.

6.1.1.1 Anesthésiologie

La Chambre est amenée à se pencher sur une proposition, déposée par le Dr M. Battaglia, présentée au nom de l'ASMAC, s'opposant à ce que la durée de la 
formation postgraduée en anesthésiologie passe de cinq à six ans.

Au vote, la proposition du Comité central (six ans) est acceptée par 110 voix contre 39, avec 18 abstentions, ce qui par voie de conséquence signifie le rejet de la proposition de l'ASMAC.

6.1.1.2 Chirurgie plastique, reconstructive et esthétique

Pro memoria, on notera ici que ce tractandum a été retiré de l'ordre du jour à la demande même de la société de discipline; celle-ci avait en effet constaté que le qualificatif "esthétique» qu'elle voulait ajouter à l'intitulé du titre de spécialiste ne figure pas non plus dans les titres de spécialiste étrangers correspondants.

\subsubsection{Création de titres de spécialiste}

6.1.2.1 Neuroradiologie; décision de la CFP du 11 mai 2000

Le Dr M. Giger, nouveau président de la CFPC, introduit la problématique que nous présentons en résumé.

Le différend qui divise la Société suisse de radiologie médicale (SSRM) de la Société de neuroradiologie réside dans le fait que la première postule la création de formations approfondies pour la neuroradiologie, alors que la seconde demande la création d'un titre spécifique pour sa discipline, en faisant valoir (son représentant, le Dr 0. Schubiger, le rappellera devant la Chambre) qu'il ne serait plus possible de couvrir les deux domaines et que l'on pourrait craindre que cette spécialité, si elle était dépourvue de titre, disparaisse. La CFPC et la Conférence des présidents proposent à la Chambre médicale la création de deux formations approfondies, l'une en "neuroradiologie diagnostique", l'autre en "neuroradiologie interventionnelle».

Au cours de la discussion nourrie qui se développe sur le sujet et qui voit les partisans des deux thèses en présence croiser le fer, le Prof. F. Follath (SSMI) soumet une proposition tendant à ce que les deux sociétés reprennent les discussions en vue de présenter à une prochaine Chambre médicale un programme de formation postgraduée permettant l'octroi d'un double titre en radiologie et neuroradiologie. Le Dr M. Giger s'oppose à cette proposition en évoquant le danger qu'une telle tergiversation représenterait pour une grande partie des autres domaines de la réglementation postgraduée en radiologie (radiologie pédiatrique, radio-oncologie) et en déclarant qu'il est impérieux que la Chambre médicale tranche aujourd'hui la controverse. Avant que l'on passe au vote, le Prof. P. Schnyder (CHUV), en sa qualité de titulaire d'une chaire de radiologie et de représentant suisse dans les plus hautes instances européennes de la spécialité, s'oppose résolument à la proposition de la Société suisse de neuroradiologie, en faisant valoir, entre autres, les conséquences financières que la création d'un nouveau titre en neuroradiologie entraînerait pour les établissements de formation, les dangers de sécession qu'il impliquerait dans la discipline et le fait que pour l'instant seuls deux pays européens (Suède et Portugal) connaissent un tel titre.
Au vote, la proposition du Prof. F. Follath est rejetée par 130 voix contre 24, avec 4 abstentions.

On peut alors passer au vote sur la proposition $d u$ CC. Par 147 voix contre 14, les délégués optent pour la création de deux formations approfondies et, par voie de conséquence, à l'admission de la Société suisse de neuroradiologie dans la CFPC.

\section{Sous-disciplines pédiatriques}

Il s'agit, ici aussi, de trancher entre les deux volets de l'alternative, titre de spécialiste ou formation approfondie. Ainsi que le précise le Dr H. H. Brunner, l'option retenue doit valoir pour toutes les sous-disciplines énumérées et il n'est pas question de traiter les unes différemment des autres. En conséquence, il propose que l'on vote sur toutes ces sous-spécialités «in globon à moins que la discussion ne soit demandée sur une sous-spécialité particulière.

Après un débat où les thèses en présence furent tour à tour défendues, notamment par les représentants des différentes sociétés gravitant dans le domaine de la pédiatrie qui plaidèrent pour la création de titres de spécialiste, on passe au vote. Le président constate au préalable que personne ne s'oppose à la procédure d'un vote "in globo" pour toutes les sousdisciplines concernées. La Chambre médicale se prononce en faveur de la solution des formations approfondies par 114 voix contre 43, avec 7 abstentions.

En conséquence, cette solution s'appliquera désormais aux sous-spécialités suivantes:

6.1.2.2 Endocrinologie-diabétologie pédiatrique

6.1.2.3 Gastroentérologie pédiatrique

6.1.2.4 Cardiologie pédiatrique

6.1.2.5 Néphrologie pédiatrique

6.1.2.6 Neurologie pédiatrique

6.1.2.7 Onco-hématologie pédiatrique

6.1.2.8 Pneumologie pédiatrique

\section{Autres programmes de formation}

Les programmes de formation postgraduée qui suivent n'ont été discutés que dans la mesure où ils ont fait l'objet d'une contre-proposition écrite à la proposition du CC (tel fut le cas pour le point 6.1.2.13); dans le cas contraire, les propositions du CC sont considérées comme acceptées. La Chambre médicale ne s'oppose pas à ce mode de procéder.

En conséquence, cette solution s'applique sans discussion au vote particulier aux spécialités suivantes:

6.1.2.9 Allergologie et immunologie clinique: création d'un titre de spécialiste FMH.

6.1.2.10 Médecine du travail: création d'un titre de spécialiste FMH.

6.1.2.11 Endocrinologie-diabétologie: création d'un titre de spécialiste FMH.

6.1.2.12 Hématologie: création d'un titre de spécialiste $\mathrm{FMH}$.

6.1.2.13 Médecine intensive: La Chambre doit se pencher ici sur une proposition présentée par l'ASMAC qui s'oppose à la création d'un titre, estimant que celui-ci n'a pas de sens, compte tenu de la vaste for- 
mation postgraduée de base que cette spécialité requiert dans d'autres disciplines cliniques. Le Dr R. Stocker, pour la Société suisse de médecine intensive, appuyé par le Dr B. Meister (anesthésiologie), plaide pour l'octroi du titre, évoquant l'effectif respectable des «intensivistes» (250 et 30 en formation postgraduée), les 60000 interventions spécifiques par année et le souci de ne pas perdre le personnel infirmier attaché aux 60 services de médecine intensive.

On passe au vote non sans que le président $\mathrm{H}$. $\mathrm{H}$. Brunner ait au préalable expliqué que ceux qui refuseraient la proposition du CC (création d'un titre de spécialiste en médecine intensive) seraient considérés comme soutenant la création d'une formation approfondie, conformément à la proposition de l'ASMAC.

$A u$ vote, la proposition du CC est rejetée par 78 voix contre 65, avec 9 abstentions. Il s'ensuit que les délégués se sont prononcés majoritairement pour la création d'une formation approfondie.

\section{Remarque importante:}

Sitôt le résultat connu, le Dr R. Stocker soulève la question des formations approfondies en relevant qu'il est impossible de créer des formations approfondies pour 7 domaines de disciplines différentes. Le Dr R. Salzberg reconnaît l'existence d'un réel problème. Il faudra sans doute remettre l'ouvrage sur le métier à la CFPC, voire au CC, et repasser éventuellement devant la Chambre médicale. Dont acte.

6.1.2.14 Pharmacologie et toxicologie clinique: création d'un titre de spécialiste FMH.

6.1.2.15 Néphrologie: création d'un titre de spécialiste FMH.

6.1.2.16 Oncologie médicale: création d'un titre de spécialiste FMH.

6.1.2.17 Pneumologie: création d'un titre de spécialiste FMH.

6.1.2.18 Radiologie: la Chambre a été appelée à trancher ici un différend portant sur la durée de la formation postgraduée, 6 ans comme le proposait le CC ou 5 ans seulement comme le demandait l'ASMAC. $A u$ vote, la proposition du CC l'emporte par 100 voix contre 40, avec 14 abstentions.

6.1.2.19 Médecine nucléaire: création d'un titre de spécialiste FMH.

6.1.2.20 Radio-oncologie/radiothérapie: une controverse identique sur la durée de la formation postgraduée doit également être tranchée entre les 6 ans préconisés par la proposition du CC et les 5 ans demandés par l'ASMAC.

$A u$ vote, la proposition du CC l'emporte par 116 voix contre 25, avec 11 abstentions. La création du titre de spécialiste FMH n'a pas été mise en cause.

\subsection{Création de formations approfondies}

6.1.3.1 Neuroradiologie diagnostique (pour la radiologie): création d'une formation approfondie et admission de la société de discipline dans la CFPC.

6.1.3.2 Neuroradiologie interventionnelle (pour la radiologie): création d'une formation approfondie et admission de la société de discipline dans la CFPC.
6.1.3.3 Radiologie pédiatrique (pour la radiologie): création d'une formation approfondie et admission de la société de discipline dans la CFPC.

6.1.3.4 Médecine de la reproduction et gynéco-endocrinologie (pour la gynécologie et obstétrique): cet objet s'inscrit dans un contexte particulier méritant quelques explications. Le Conseil fédéral (CF) doit prochainement mettre en vigueur la loi fédérale sur la procréation assistée adoptée par le Parlement en décembre 1998. Cette loi prévoit que les médecins qui pratiquent ces techniques doivent obtenir une autorisation. Plutôt que ce soit la Confédération qui fixe les exigences, une solution se dessine pour que ce soit la FMH qui les définisse dans le cadre de sa RFP, par la création anticipée d'une formation approfondie. L'Office fédéral de la Justice propose au CF de faire référence à la réglementation de la FMH dans l'ordonnance d'application. Cela explique la pression du temps à laquelle nous sommes soumis dans cette affaire.

Cette pression du temps a paru inadmissible à l'ASMAC qui, dans un premier temps et sans s'opposer au principe même de la création d'une formation approfondie, avait déposé une proposition de non-entrée en matière. Elle fut retirée en cours de débat, après que l'assurance fut donnée que le programme (non contesté) de la formation approfondie pour ce domaine, présenté dans le cadre du programme pour le titre de gynécologie et obstétrique en juin 1999, n'avait subi aucune modification.

La Chambre peut ainsi accepter la proposition $d u$ $C C$ de créer en principe une formation approfondie en médecine de la reproduction et en gynéco-endocrinologie. Il est pris acte de ce que les questions de détail techniques qui pourraient résulter de cette décision anticipée par rapport au titre de base encore inchangé devront être réglées entre la SDM concernée, la CFPC et le CC.

\subsubsection{Création d'attestations de formation complémentaire}

6.1.4.1 Médecine de plongée (SSMSH): création d'une attestation de formation complémentaire en médecine de plongée (SSMSH).

\subsubsection{Création de certificats d'aptitude technique}

6.1.5.1 Radiologie à fortes doses: création de certificats d'aptitude technique pour les examens radiologiques à fortes doses.

6.1.5.2 Laboratoire (CMPR): création d'un certificat d'aptitude technique.

6.1.5.3 Laserthérapie de la peau et des muqueuses cutanées (FMS): création d'un certificat d'aptitude technique.

\subsection{Révision de la Réglementation}

pour la formation postgraduée (RFP)

Avant que l'on passe au vote sur l'ensemble de la RFP, la Chambre est amenée à se pencher sur une proposition du Dr M. Battaglia, déposée au nom de l'ASMAC, demandant que les commissions de recours se composent de 5 membres, à savoir un juriste in- 
dépendant, 2 membres du CC, un membre d'une SCM et un membre de l'ASMAC.

On fait valoir, du côté du CC, les bonnes expériences faites avec une commission de 3 membres et le souci d'une administration à moindres frais.

Au vote, la proposition $d u$ CC sur ce point précis est acceptée par 115 voix contre 19, avec 5 abstentions.

A la demande du Dr R. Salzberg, la Chambre procède à un vote formel sur la RFP dans son ensemble, après toutes les décisions de détail la concernant, nonobstant qu'à partir du $1^{\mathrm{er}}$ janvier 2001, ce sera une loi fédérale qui prendra le relais.

$A u$ vote, la RFP est littéralement plébiscitée par 138 voix contre 2 , avec 6 abstentions.

6.3 Loi sur l'exercice des professions médicales: octroi de titres fédéraux de formation postgraduée à des médecins sans titre

Le législateur fédéral a prévu à l'article 24, al. 3, de la loi sur l'exercice des professions médicales l'octroi, à tous les médecins dépourvus de titre, d'un titre postgrade correspondant à leur formation pratique et théorique parmi les titres figurant dans l'ordonnance d'exécution à la loi. Cette disposition s'avère nécessaire dès lors que l'exercice de la profession médicale à titre indépendant ne sera plus possible sans titre postgrade fédéral. Pour ce faire, l'ordonnance propose deux variantes entre lesquelles, bien que la décision finale appartienne au Conseil fédéral, le CC souhaite disposer de l'avis consultatif de la Chambre.

La variante I, comprenant elle-même deux sousvariantes, postule la vérification complète du curriculum de formation. Dans la sous-variante 1, une année de formation postgrade manquante pourrait être remplacée par une année d'activité dans un cabinet médical, alors que la sous-variante 2 permettrait de compenser jusqu'à trois années de formation postgrade manquante par une activité en cabinet.

La variante II, tenant compte des vœux de la SSMG, faciliterait l'octroi d'un titre de spécialiste en médecine générale pour peu que le candidat puisse attester de deux années de formation postgrade valide.

Le Dr R. Hohendahl, de la Foederatio Medicorum Practicorum (FMP), demande à la Chambre médicale de charger le CC de continuer à négocier (avec l'OFSP) au sens de la sous-variante 2 .

Au terme d'un débat nourri, la proposition de la FMP est soumise au vote: elle est rejetée par 91 voix contre 37, avec 9 abstentions.

\section{Admission de la Société suisse des chiropraticiens en tant que $4^{\mathrm{e}}$ société fondatrice de la Caisse de compensation des médecins, dentistes et vétérinaires (Caisse AVS $n^{\circ}$ 28)}

Ce point de l'ordre du jour est traité en présence de Mme Franziska Iseli, notaire à Berne.

Le secrétaire général explique brièvement le contexte de la proposition. La parole n'est pas demandée. Le président demande alors à l'assemblée, au nom du CC de la FMH, d'accepter la demande de la Société suisse des chiropraticiens d'être admise à titre de $4^{\mathrm{e}}$ société fondatrice dans la Caisse de compensation AVS $n^{\circ} 28$, à partir du $1^{\mathrm{er}}$ janvier 2001. Mise au vote, cette proposition obtient 161 voix pour, zéro contre, avec 2 abstentions. La majorité qualifiée des $3 / 4$ des suffrages exprimés (123) est atteinte et la proposition est ainsi acceptée.

\section{Code de déontologie $\mathrm{FMH} /$ révision partielle}

Dans une proposition présentée par le Prof. L. von Laer au nom de la Société bâloise de médecine, il est demandé que l'éventail des sanctions prévues par l'article 47, al. $1^{\mathrm{er}}$, du Code de déontologie de la FMH du 12 décembre 1996 soit enrichi, à partir du $1^{\text {er }}$ janvier 2001, par la possibilité d'encourager et de soutenir les médecins concernés au moyen d'une supervision (lit. h nouvelle). Le proposant fait valoir qu'une telle manière de faire serait dans certains cas (abus sexuels de patient[e]s) plus efficace que des sanctions purement pénales.

Le président $\mathrm{H}$. H. Brunner ne s'oppose pas à cette proposition que la Chambre médicale accepte tacitement. Elle est donc transmise aux services compétents pour exécution.

\section{Loi sur les produits thérapeutiques (information orale)}

La loi qui a été traitée au Conseil national sans que nos desiderata aient été retenus se trouve actuellement dans la commission ad hoc du Conseil des Etats: c'est ici qu'il s'agira donc de faire valoir nos postulats (solution libérale pour la distribution des médicaments, en la confiant à toutes les personnes - médecins inclus - qui en ont la compétence et solution raisonnable en ce qui concerne la stérilisation). C'est le lieu de rappeler ici que le CC a reçu la compétence de la Chambre médicale de lancer une initiative si l'évolution du dossier le rendait nécessaire. Par ailleurs, il nous reste également la possibilité d'un référendum contre la loi. On retiendra enfin la suggestion du Dr A. Haefeli demandant au CC, à l'intention des SCM, un mémoire résumant ce qui, du point de vue de la FMH, doit absolument se trouver dans cette loi; le président $\mathrm{H}$. $\mathrm{H}$. Brunner en prend bonne note au nom du CC. Affaire à suivre.

\section{Obligation de contracter (information orale)}

Le Dr R. Streit, au nom de la Société des médecins $d u$ canton de Berne, demande dans une proposition ad point 10 de l'ordre du jour que soit mené «un débat de fond sur la situation de la politique de la santé, en tenant compte des déclarations de Mme la Conseillère fédérale R. Dreifuss au Conseil des Etats selon lesquelles la valeur du point devrait être de 80 cts. Il s'agit aussi d'évoquer la proposition du Département 
fédéral de l'intérieur concernant la levée de l'obligation de contracter dans l'assurance sociale de base». Cette proposition est acceptée à la quasi-unanimité, contre quelques voix isolées.

Un long débat s'instaure alors qui illustre, comme s'il en était vraiment besoin, le degré d'exaspération auquel est parvenu le corps médical rassemblé au sein de la FMH. Des propos fort vifs sont tenus à l'adresse de la Conseillère fédérale, Mme R. Dreifuss, pour sa nouvelle tentative de pression sur nos débats ainsi que pour être sortie de la réserve qui devrait être la sienne en tant que future "juge de dernière instance» appelée à se prononcer sur le TarMed, au point de perdre toute crédibilité aux yeux de la Chambre médicale quant à son impartialité.

En ce qui concerne plus particulièrement la levée de l'obligation de contracter, la Chambre est prête à convenir d'une limitation de l'obligation de contracter dans le but de laisser les meilleurs médecins travailler au service des patients de l'assurance sociale et rappelle ses propositions en la matière adressées à l'OFAS et au Concordat des assureurs-maladie suisses. En revanche, la FMH ne reculerait pas devant un référendum si la réforme de la loi était celle proposée par le Département fédéral de l'intérieur.

Pour ce qui a trait au TarMed et sans préjuger des décisions ponctuelles encore à prendre sous point 11 ci-dessous, les délégués délivrent l'instruction aux délégués aux négociations de ne pas convenir d'une valeur du point inférieure à Fr. 1.- lors des négociations finales.

Au terme de la discussion, le président H. H. Brunner en résume les points principaux et informe la Chambre qu'il en sera établi un communiqué de presse. La Chambre accepte sans discussion aussi bien le résumé établi par le président que le principe d'un communiqué.

NB: Cette décision fut exécutée le soir même par la publication de deux communiqués de presse, "Le corps médical contre les tentatives d'intimidation du DFI" et "Suppression de l'obligation de contracter: la FMH envisage le référendum».

\section{TarMed}

Le président $\mathrm{H}$. H. Brunner est amené à fournir réponse aux représentants de la FMS (C. Krayenbühl et F. A. Bernath) sur diverses questions relatives aux études de terrain (on a renoncé aux études-pilotes, vu les frais considérables à envisager), aux études de panier type (cela doit rester des instruments de pilotage à usage interne) ainsi que sur l'expertise juridique indépendante concernant les droits et obligations des divers intervenants dans l'introduction du TarMed (réticence des juristes compétents à s'engager et intérêt plus que mesuré au sein du TarMed). Les perspectives sont meilleures en ce qui concerne le mandat à confier à un institut neutre étranger sur la question de l'établissement de la structure tarifaire selon les règles de la gestion d'entreprise.
Les interpellants/proposants se déclarent satisfaits de ces réponses.

\subsection{Révision 2000}

La Chambre prend connaissance des résultats de la révision 2000 ainsi que du commentaire correspondant qui ne se prête guère à un exposé dans le BMS, c'est-à-dire sur la place publique.

\subsection{Modèle tarifaire de la FMS}

La Chambre prend également connaissance du commentaire écrit à propos de cet objet ainsi que des explications complémentaires fournies en cours de débat par le président, explications qui valent réponse à une proposition de la FMS $\left(\mathrm{n}^{\circ} 2\right.$ ad point 11.2 de l'ordre du jour) demandant que le modèle FMS soit introduit avant la mise en vigueur du TarMed.

\subsection{Structure tarifaire TarMed alpha 3.0/ \\ Pré-contrat}

On retiendra ici que la version alpha 3.0 de la structure tarifaire est en voie d'élaboration et qu'elle se base sur la révision 2000 et contiendra toutes les propositions et modifications traitées jusqu'au 27 mai 2000. Cette version constituera la base des négociations pour le tarif médical AA/AM/AI, pour le catalogue des prestations hospitalières ainsi que pour les tarifs cantonaux. Une version alpha 3i sera publiée ultérieurement; elle ne prendra en compte que des modifications de forme et rédactionnelles. Elle sera envoyée à tous les membres de la FMH sous forme de CD-ROM et fera l'objet d'une votation générale. Les propositions nous étant parvenues après le 15 avril 2000 sont renvoyées à la $1^{\text {ère }}$ révision de la version 3.0 .

Une proposition ( $\mathrm{n}^{\circ} 2$ ad point 11.3) de la Société suisse de psychiatrie et de psychothérapie ainsi que de la Société suisse de psychiatrie et psychothérapie d'enfants et d'adolescents prie le CC «de prendre les mesures appropriées pour que l'on reprenne en considération les buts initiaux du GRAT, à savoir une meilleure prise en compte des prestations intellectuelles et émotionnelles [et] la réduction des différences non justifiées entre les revenus des diverses disciplines médicales». Après explications des proposants et réponse du président $\mathrm{H}$. H. Brunner, cette proposition est acceptée tacitement par les délégués.

Une autre proposition ( $\mathrm{n}^{\circ} 3$ ad point 11.3) émanant conjointement de la SSMG, de la SSMI, de la $S S P$ et du Collège de médecine de premier recours tend à ce que «le chiffre 21 des interprétations générales de la version TarMed alpha 2.2. (ou chiffre 85 suivant la version) [soit] modifié impérativement». La proposition, trop détaillée pour être citée ici in extenso, vise en substance à maintenir la possibilité de facturer en sus certains matériaux d'utilité courante dans les cabinets de médecine de premier recours, sans quoi de nombreuses prestations utiles et peu coûteuses ne seraient plus fournies qu'à l'hôpital. Les exemples précis de ces aberrations pourraient être communiqués à qui de droit. 
L'affaire est d'importance, les intervenants du côté des sociétés proposantes laissant entendre que le maintien de l'interprétation critiquée est de nature à compromettre une acceptation du TarMed en votation générale. Dont acte!

\subsection{Convention-cadre CAMS-FMH}

Après que le Dr A. Haefeli, président du "G 7», ait rappelé les activités de ce groupe de travail et les résultats intermédiaires déjà obtenus, et après un débat largement utilisé, la Chambre médicale, conformément à la proposition $d u C C$, prend connaissance et approuve tacitement la convention-cadre CAMSFMH et attribue à la délégation aux négociations le mandat de poursuivre les pourparlers jusqu'à leur conclusion, en se basant sur les conclusions et les décisions de la Chambre médicale, et de présenter le projet définitif de la convention-cadre lors de la prochaine séance de la Chambre médicale. On notera encore ici, à la demande expresse du Dr L. Dubs, de la Société suisse d'orthopédie, que le sigle EBM utilisé dans ce contexte ne désigne pas l'Evidence-based Medecine, mais bien les «Einheitliche Bemessungsmassstäbe». Dont acte.

\subsection{Concept de neutralité des coûts CAMS-FMH} La proposition du CC sur cet objet tend à ce que la Chambre approuve le concept de neutralité des coûts (ci-après: NC) CAMS-FMH, en intégrant, le cas échéant, les décisions de la Chambre médicale, avec mandat à notre délégation aux négociations de mener les pourparlers jusqu'à leur conclusion et de présenter le projet définitif dudit concept lors de la prochaine séance de la Chambre médicale. Plusieurs propositions furent déposées sur cet objet. La première $\left(\mathrm{n}^{\circ} 2 \mathrm{ad}\right.$ point 11.5) qui fut présentée le fut par le Dr R. Streit, pour la Société des médecins du canton de Berne; elle comprend 5 points. Le point 1 constate que la NC répartie en secteurs partiels (cantonal, ambulatoire, ambulatoire hôpital et hospitalier) qui devraient être neutres en termes de coûts n'est pas conforme au tarif d'économie d'entreprise prescrit par la loi. Il s'agit de l'indiquer haut et fort. Le point 2 exige que la NC soit définie et limitée clairement. Le terme NC signifie que les coûts par assuré ne doivent pas être modifiés par l'introduction de la structure tarifaire. Toutes les influences non structurelles ayant un effet sur le tarif doivent autant que possible être enregistrées et imputées positivement ou négativement à la somme de la NC. Le point 3 concerne l'angle alpha: défini comme valeur moyenne nationale, il devra être adapté dans les cantons vu que les transferts entre les secteurs neutres en matière de coûts peuvent varier d'un canton à l'autre. Quant au point 4 , il demande que la phase de réglage suivant l'introduction du TarMed soit limitée à 2 ans au maximum. Le point 5 demande enfin que les concepts présentés soient remaniés et évalués quant à leur contradiction. Au vote, les points 1, 2, 3 et 5 sont acceptés chaque fois à une écrasante majorité. En ce qui concerne le point 4, celui-ci est accepté en ce qui concerne la NC proprement dite, le président $\mathrm{H}$. H.
Brunner faisant toutefois observer que la Chambre médicale avait précédemment accepté le principe d'une phase de convergence de 5 ans. La seconde proposition ( $\mathrm{n}^{\circ} 3$ ad point 11.5), émanant également du Dr R. Streit (BE), en trois points, rappelle que la Chambre médicale a approuvé la structure tarifaire calculée sur une valeur du point tarifaire de Fr. 1.- et que donc une valeur du point de Fr. 0.80 est expressément refusée (point 1), que Mme la Conseillère R. Dreifuss, par sa déclaration au Conseil des Etats sur la valeur du point avant la publication du résultat des négociations, a fait preuve d'une attitude partiale envers le corps médical (point 2) et qu'il ne devrait pas y avoir d'approbation définitive des résultats des négociations tant qu'elle n'aura pas retiré ses affirmations (point 3). Au vote, cette proposition est acceptée à une très forte majorité.

La troisième proposition ( ${ }^{\circ} 4$ ad point 11.5) émane de la Société de médecine du canton de Fribourg. Elle demande en substance que la Chambre médicale n'accepte l'introduction du TarMed que lorsque le concept de la neutralité des coûts sera signé par toutes les parties concernées (CAMS, Commission des tarifs médicaux LAA, CDS et $\mathrm{H}+$ ), car il est impérieux qu'une seule méthode de calcul et d'analyse soit utilisée. Le président $H$. H. Brunner se saisit de cette proposition et promet de la défendre dans les négociations. La proposition est ainsi acceptée tacitement.

Une quatrième proposition ( $\mathrm{n}^{\circ} 5$ ad point 11.5) présentée par la FMS est retirée par ses auteurs au profit des propositions des Drs R. Streit et E. Steinmann. La proposition de ce dernier $\left(\mathrm{n}^{\circ} 6\right.$ ad point 11.5), présentée au nom de la Société de médecine $d u$ canton de Lucerne, en se basant sur des décisions prises lors de précédentes Chambres médicales, demande que le concept de NC remplisse les points suivants: le but du concept de NC est l'introduction neutre du point de vue des coûts du nouveau tarif. La phase de la stricte NC doit donc être réduite au minimum; l'angle alpha doit être défini sur le fond de telle façon qu'il ne devienne pas l'objet de négociations ultérieures; le principe, le but et le mécanisme de la convergence (qui doit être terminée au plus tard dans 5 ans) doivent être réglés strictement dans le concept; les conditions-cadres pour l'implémentation du concept (liste des analyses, tarif de physiothérapie, réglementation de la distribution des médicaments, obligation de contracter, etc.) font partie intégrante du concept; les voies de droit, resp. l'exigence d'un arrêté fédéral urgent ne sont pas dans les compétences des partenaires contractuels et ne sauraient donc faire partie du concept. Le concept, en général et en détail, doit donc être modifié pour tenir compte de ces exigences. Le président $\mathrm{H}$. H. Brunner insiste pour que les conditions-cadres qui seront la garantie du succès du fonctionnement de ce concept soient exhaustivement énumérées. Ce message aux politiciens est indispensable. Cela dit, il doit s'opposer au passage concernant l'arrêté fédéral urgent, car les choses sont déjà réglées dans la dernière version du concept (non encore en possession des délégués). 
Le président constate en conclusion sur cet objet que la proposition du Dr E. Steinmann est acceptée tacitement, avec la précision apportée par le Dr H. H. Brunner lui-même.

11.6 Concept concernant la valeur intrinsèque La Chambre médicale accepte la proposition $d u$ CC d'approuver le concept concernant la valeur intrinsèque, dans la version du 4.6.00, donc dans une version qui ne s'appuie pas sur la RFP actuelle. Le président H. H. Brunner précise que rien ne s'oppose toutefois à ce que l'on rediscute ultérieurement avec les SDM les critères qualitatifs d'attribution de la valeur intrinsèque.

\subsection{Convention-cadre AA/AM/AI}

La Chambre médicale accepte la proposition $d u$ CC d'approuver la convention-cadre AA/AM/AI dans sa version 4.

11.8 Concept de neutralité des coûts AA/AM/AI Pas encore de décision car pas encore de document.

11.9 Valeur initiale du point tarifaire AA/AM/AI La Chambre médicale prend connaissance du commentaire à ce propos, étant entendu que la décision définitive demeure réservée à la prochaine Chambre médicale. Le Dr B. Meister, de la Société suisse d'anesthésiologie, demande que l'on vote sur sa proposition qui tend à ce que la valeur du point initiale soit fixée à Fr. 1.-. Au vote, la proposition du Dr B. Meister recueille 125 voix contre 3 et 13 abstentions.

11.10 Agenda pour l'introduction des tarifs TarMed La Chambre médicale approuve, par 138 voix, sans opposition ni abstention, l'agenda proposé par le CC qui prévoit que les négociations soient menées de façon à permettre l'introduction du tarif AA/AM/AI entre le $1^{\text {er }}$ janvier et le $1^{\text {er }}$ juillet 2001. Il s'agira en particulier de négocier la/les valeur(s) initiale(s) du point tarifaire AA/AM/AI d'ici à la fin août 2000. Les travaux et négociations concernant l'introduction $\mathrm{du}$ TarMed dans les tarifs cantonaux doivent être organisés de façon à permettre une introduction réglementaire au $1^{\text {er }}$ juillet 2001. Le CC élabore un scénario de rechange permettant, suivant l'évolution de la scène politique, une introduction au $1^{\mathrm{er}}$ janvier 2001. Les conventions-cadres AA/AM/AI ainsi que les conventions-cadres CAMS-FMH, avec leurs annexes, doivent être soumises à une Chambre médicale extraordinaire pour approbation. Le CC fixe la date correspondante de manière à garantir la possibilité d'une votation générale.

L'acceptation de ce calendrier entraînera peut-être la convocation d'une Chambre médicale qui ne pourra pas être précédée, comme les statuts le prévoient, d'une Conférence des présidents à tenir dans les six semaines avant. La Chambre est dûment informée par le président de cette suspension des statuts due à la loi de la nécessité. Il est constaté formellement qu'aucune opposition ne se manifeste contre cette manière de faire.

\subsection{Votation générale}

La Chambre médicale accepte, par 137 voix, sans voix contre ni abstention, la proposition $d u$ CC prévoyant l'organisation d'une votation générale avant l'entrée en vigueur des tarifs TarMed dans le domaine AA/ AM/AI ainsi que dans celui de la LAMal. Feront l'objet de la votation générale, la structure tarifaire TarMed alpha 3i, la convention-cadre AA/AM/AI, avec ses annexes et la convention-cadre CAMS-FMH, avec ses annexes. La votation générale, qui n'aura lieu que si tous ces points auront été approuvés par la Chambre médicale, devra avoir lieu directement après que la Chambre se sera prononcée sur lesdits points.

\subsection{Transfert électronique des données}

Ce point ne donne pas lieu à décision.

\section{Concept PR de la FMH}

Vu l'heure avancée, une discussion n'a plus pu se tenir sur ce document émanant de la plume du président. Ce dernier invite les délégués à lui faire part de leurs éventuelles remarques et suggestions dont il sera dûment tenu compte.

\section{Divers}

A retenir sous cette rubrique:

- que la FMH ne reste pas inactive sur le terrain des "Call Centers» et fera connaître sa position en tant opportun.

- que l'ORDINE, par la voix du Dr A. Sury, invite la Chambre médicale à tenir ses assises l'année prochaine au Tessin, à l'occasion du centenaire de la FMH. Le président H. H. Brunner accepte positivement cette offre qui devra toutefois encore être étudiée.

Il est près de 17 heures lorsque la session est levée par le président qui salue l'assiduité mise par les délégués à venir à bout d'un ordre de jour impressionnant et qui remercie tous les collaboratrices et collaborateurs du secrétariat général ayant contribué au bon déroulement de ces deux jours de séance, avec mention particulière au service de traduction et aux interprètes. 